\title{
CODEBOOK DESIGN STRATEGIES FOR MMO-OFDM DOWNLINK SYSTEMS WITH ZF-BF
}

\author{
Najoua ACHOURA ${ }^{1}$ and Ridha BOUALLEGUE ${ }^{2}$ \\ ${ }^{1}$ Department National Engineering School of Tunis, \\ Innov'Com Laboratory, Tunisia \\ ${ }^{2}$ High School of Communication of Tunis, SUP'COM, \\ Innov'Com Laboratory, Tunisia
}

\begin{abstract}
Using the Channel State Information (CSI) at the transmitter is fundamental for the precoder design in Multi-user Multiple Input Single Output (MU-MISO-OFDM) systems. In Frequency Division Duplex (FDD) systems, CSI can be just available at the transmitter through a limited feedback channel [1], where we assume that each user quantizes its channel direction with a finite number of quantization bits. In this paper, we consider a scalar quantization (SQ) scheme of the Channel Direction Information (CDI). Although vector quantization (VQ) schemes [2], [3] still outperform this scalar scheme in terms of quantization error and Sum rate, the former scheme suffers from an exponential search complexity and high storage requirements at the receiver for high number of feedback bits.
\end{abstract}

\section{KEYWORDS}

MIMO-OFDM, zero-forcing beamforming, $R V Q$, Scalar Quantization

\section{INTRODUCTION}

Currently, higher data rate is preferred to supply high quality multi-media services. Multipleinput multiple-output (MIMO) technology has attracted much more attentions since MIMO wireless channels, created by exploiting antenna arrays at transmitter and receiver, promises high capacity and high quality wireless communication links. It is well-known that with full channel state information (CSI) at the transmitter (CSIT), employment of precoding techniques can improve its capacity [4], [5]. This implies that the transmitter requires some form of knowledge on the wireless channel conditions. In time division duplexing (TDD) systems, reciprocity can be employed to explore CSI and make CSI available at transmitter. However, employing reciprocity in frequency division duplexing (FDD) systems has been impossible since the forward and reverse links in FDD generally have highly uncorrelated channels. The adaptation of feedback makes instantaneous CSIT possible. In practical systems, the receiver estimates the channel conditions based on the pre-defined reference signals known by both transmitter and receiver. After channel estimation, the receiver sends the estimated channel information back to the transmitter side, and the transmitter uses this information to adapt the forward link transmission. The adoption of feedback can improve the system performance, such as increasing capacity and reducing the failure rate of data transmission, which cannot be handled by the receiver alone. However on the other hand, the feedback information itself occupies some frequency resource 
and decreases the spectrum efficiency of system. That's why limited feedback is necessary in practical systems over limited bandwidth feedback channels. There are many works dealing with limited feedback [6]. One of them is generating limited feedback based on the pre-defined codebook, which is known by both transmitter and receiver. Once the receiver obtained the CSI by channel estimation, it checks the codebook to figure out the quantization partitions, and represent all the channel information located in these partitions by the corresponding codewords. After finding those codewords from codebook, the receiver sends the codebook index corresponding to these codewords back to the transmitter instead of sending full CSI back. At the transmitter side, the transmitter chooses the codewords from the identical codebook based on the feedback information from receiver. Usually, the systems suffer lower burden to send codebook index rather than feedback full CSI.

In this situation, the strategies of generating codebook become the key issues. In this paper, we propose two codebook generation strategies for MISO beamforming systems. The first strategy is based on scalar quantization, which has lower computational complexity compared to high dimensional (more than 2 dimension) vector quantization strategies.

\section{SYSTEM MODEL}

The downlink or broadcast system is described as follows. The base station with $\mathrm{N}_{\mathrm{t}}$ antennas transmits data simultaneously to $\mathrm{N}_{\mathrm{t}}$ active users chosen from a total of $\mathrm{U}$ users, each with one receive antenna. The base station separates the multiuser data streams by beamforming, i.e. assigning a beamforming vector to each of the $\mathrm{N}_{\mathrm{t}}$ active users. The beamforming vectors $\left\{w_{n}\right\}_{n=1}^{N t}$ are selected from multiple sets of unitary orthogonal vectors following the beam and user selection algorithm. Equal power allocation over scheduled users is considered. The received signal of the $\mathrm{u}^{\text {th }}$ scheduled user is expressed as:

$$
y_{u}=\sqrt{\frac{P}{N t}} h_{k}^{*} \sum_{n \in A} w_{n} x_{n}+v_{u} \quad u \in A
$$

Where we use the following notation:

$N_{t}$ :number of transmit antennas and also number of scheduled users; $h_{u}\left(\mathrm{~N}_{t} \times 1\right.$ vector $)$ downlink channel; $x_{u}$ transmitted symbol with $\mathrm{E}\left[|x u|^{2}\right]=1 ; y_{u}$ received symbol; $w_{u}\left(\mathrm{~N}_{t} \times 1\right.$ vector $)$ beamforming vector, $A$ The index set of scheduled users, $\mathrm{P}$ :transmission power;

\section{ZERO-FORCING BEAMFORMING}

Zero-forcing (ZF) decomposes the channel into several parallel scalar channels with only additive noise, and the interference is removed completely by transmit beamforming techniques[12]. Suppose a base station with Nt transmit antennas transmits information to $\mathrm{K}$ users, each user is equipped with one single receive antenna. The received signal by the kth user is

$$
\mathrm{y}_{\mathrm{k}}=\mathrm{h}_{\mathrm{k}}^{\mathrm{H}} \mathrm{v}_{\mathrm{k}} \mathrm{x}_{\mathrm{k}}+\mathrm{h}_{\mathrm{k}}^{\mathrm{H}} \sum_{\mathrm{j}=1, \mathrm{j} \neq \mathrm{k}}^{\mathrm{K}} \mathrm{v}_{\mathrm{j}} \mathrm{x}_{\mathrm{j}}+\mathrm{n}_{\mathrm{k}} \quad 1 \leq \mathrm{k} \leq \mathrm{K}
$$

ZF method transmits the signals towards the intended user with nulls steered in the direction of the other users, i.e., $\left(h_{j}^{H} v_{k}=0 \forall j \neq k\right)$. The users will receive only the desired signal without any interference because of the perfect nulling. In this case, the received data at the $\mathrm{k}^{\text {th }}$ user can be written 


$$
y_{k}=h_{k}^{H} v_{k} x_{k}+n_{k}
$$

The corresponding vector equation is

$$
y=H^{h} V x+n
$$

Therefore, if the normalized transmit beamforming vector of the $\mathrm{k}^{\text {th }}$ user is selected

$$
v_{k}^{\prime}=\frac{h_{k}^{(\dagger)}}{\sqrt{\left\|h_{k}^{(\dagger)}\right\|_{F}^{2}}}
$$

where $h(y) k$ is the $k^{\text {th }}$ column of the pseudo inverse of $H$, denoted as $H(y)$. Then it is shown that the interference can be canceled completely. In this case, the SNR of the kth user is

$$
S N R_{k}=\frac{\left|h_{k}^{H} v_{k}^{\prime}\right|^{2} p_{k}}{\sigma^{2}}
$$

\section{SCALAR QUANTIZATION}

We assume that our system is a multiuser MIMO (MU-MIMO) downlink. As shown in Figure 1, there is $\mathrm{N}_{\mathrm{t}}$ transmit antennas at base station (BS) side, and $\mathrm{Nr}$ mobile stations (MS) communicate with BS simultaneously. Each mobile system is equipped with a single antenna, and the relationship $N_{r} \leq N_{t}$ holds in this system.

Since the base station only needs the spatial direction of the channel to eliminate the interference, the channel matrix $\mathrm{H}$ is normalized and then quantized using Scalar quantization at each user. The real and imaginary parts of each complex element $h_{i j}$ in $\mathrm{H}$ are quantized to $B_{\mathrm{S}}$ bits, respectively

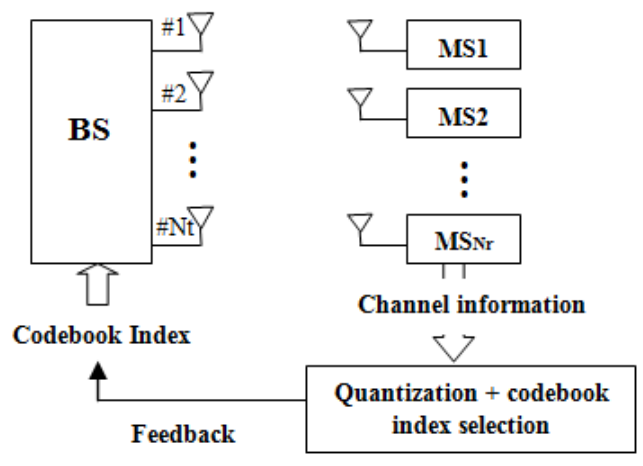

Figure 1. Block diagram of MU-MIMO system with limited feedback

Note that one bit is reserved for the sign of each of the real and imaginary parts. In [7], the quantized version of $h_{i j}$ is $h_{i j}^{\prime}=\frac{1}{2^{B} s^{-1}}\left[\frac{h_{i j}}{m}\left(2^{B_{s}-1}-1\right)\right]$, where $m$ is a scaling ratio which guarantees the real/imaginary element in the normalized channel matrix is always less than or equal to one. In particular, $m$ can be chosen as the maximum value among all real and imaginary elements of the channel matrix $H$. Therefore, the number of feedback bits needed at each user is $B=2 N_{t} N_{r} B_{s}$.

A closed-form expression for the relationship between the rate loss and the number of feedback bits is usually intractable. A feasible approach is to approximate the quantization error as a 
random variable with a given distribution, e.g., uniformly distributed in $\left[-2^{-B_{S}-1}, 2^{-B_{S}-1}\right]$. For the sake of analytical simplicity, we assume the quantization error is a Gaussian random variable with zero mean and variance $\sigma_{s}^{2}=\frac{1}{12} 2^{-2 B_{S}+2}$ [8]. As the sum rate is a function of the variance [9], by applying a similar approach as in Theorem 2 in [10], an approximation for the number of feedback bits required to maintain a performance gap of no more than $3 \mathrm{~dB}$ with respect to the case with perfect CSI is derived to be

$$
B \approx 2 N_{t} N_{r}\left(\frac{P_{d B}}{3}-\frac{1}{2} \log \frac{N_{t}}{12}\left(2^{\frac{N_{t}}{N_{r}}}-1\right)\right)
$$

Where $P_{d B}=10 \log _{10} P$ is the normalized transmit power in units of $\mathrm{dB}$.

\section{CHANNEL VeCTOR QUANTIZATION}

In [11], authors analyzed the channel capacity with perfect channel knowledge at the receiver, but with limited channel knowledge at the transmitter. Specifically, the optimal beamformer is quantized at the receiver, and the quantized version is relayed back to the transmitter. Given the quantization codebook $C=\left\{w_{1}, \ldots ., w_{2^{B}}\right\}$, which is also known a priori at the transmitter, and the channel $\mathrm{H}$, the receiver selects the quantized beamforming vector to maximize the instantaneos rate, [11]

$$
w(H)=\arg \max _{v_{j} \in V}\left\{\log \left(1+\rho\left\|H w_{j}\right\|^{2}\right)\right\}
$$

Where $\rho=1 / \sigma_{n}^{2}$ is the background signal-to-noise ratio (SNR). The (uncoded) index for the ratemaximizing beamforming vector is relayed to the transmitter via an error-free feedback link. The capacity depends on the beamforming codebook V and B. With unlimited feedback $(B \rightarrow \infty)$ the $\mathrm{w}(\mathrm{H})$ that maximizes the capacity is the eigenvector of $\mathrm{H}^{*} \mathrm{H}$, which corresponds to the maximum eigenvalue.

We will assume that the codebook vectors are independent and isotropically distributed over the unit sphere. It is shown in [12], that this RVQ scheme is optimal (i.e., maximizes the achievable rate) in the large system limit in which $\left(B, N_{t}, N_{r}\right) \rightarrow \infty$ with fixed normalized feedback $B=B / N_{t}$ and $\mathrm{N}_{\mathrm{r}}=\mathrm{N}_{\mathrm{r}} / \mathrm{N}_{\mathrm{t}}$. (For the MISO channel $\mathrm{N}_{\mathrm{r}}=1$ ). Furthermore, the corresponding capacity grows as $\log \left(\rho \mathrm{N}_{\mathrm{t}}\right)$, which is the same order-growth as with perfect channel knowledge at the transmitter. Although strictly speaking, RVQ is suboptimal for a finite size system, numerical results indicate that the average performance is often indistinguishable from the performance with optimized codebooks.

\section{SIMULATIONS RESULTS}

In Figure 2, the sum rate is plotted versus SNR for the ZF-BF technique by considering the case of a perfect CSIT in comparison with the use of vector quantization RVQ for $\mathrm{B}=8,16$ and 32 bits, the number of transmit antennas is $\mathrm{N}_{\mathrm{t}}=4$. When the $\mathrm{SNR}$ is low, the limited feedback reacts almost as well as the zero-forcing with full CSI. However, when the SNR increases, the feedback system is limited by interference and the rate converges to a threshold limit based on the amount of the multi-user interference. 


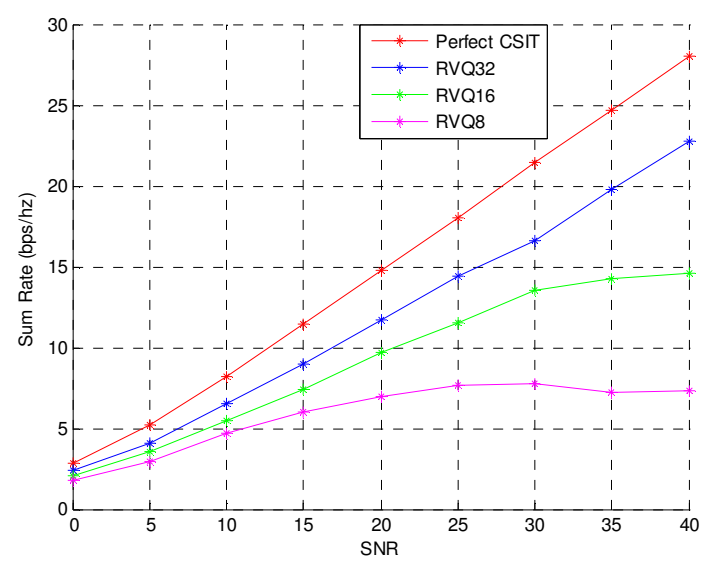

Figure 2. Comparison of the ZF-BF method using RVQ for different sizes of feedback

In Figure 3 the sum rate with RVQ quantization, as well as perfect CSI case for the same number of users (i.e. users $\mathrm{T}_{\mathrm{fb}} / \mathrm{B}$ ) are plotted as a function of $\mathrm{B}$ for a system with $\mathrm{N}_{\mathrm{t}}=4, \mathrm{~T}_{\mathrm{fb}}=300$ bits and $\mathrm{SNR}=15 \mathrm{~dB}$. We note that for $\mathrm{B} \geq 25$ the RVQ perfectly approaches the case of perfect CSIT. As a result, increase the size of feedback beyond 25 bits no sense obviously because it reduces the number of users, but does not provide a measurable benefit. In the same figure, and considering the case of a scalar quantization, although we note that it provides a sum slightly below the RVQ. However, it is still interesting to work with a large $\mathrm{B}$, which is generally the preferred operating point, even with a dictionary suboptimal quantization, and performance with scalar quantization is still competitive.

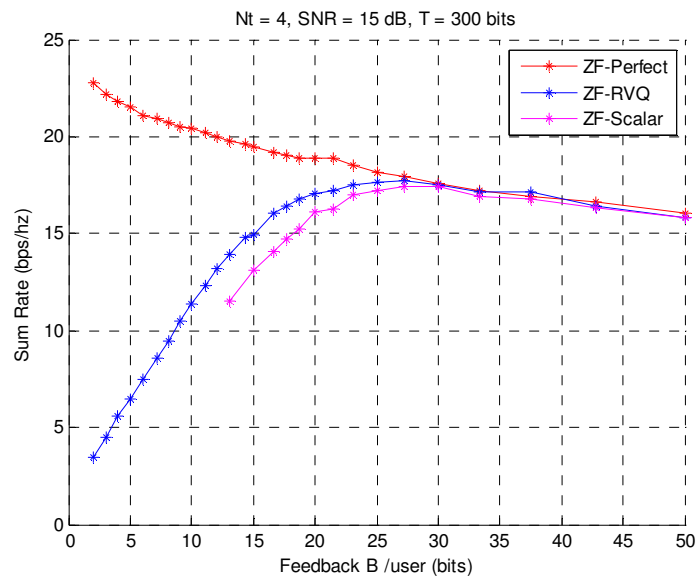

Figure 3. ZF-BF with RVQ and scalar quantization vs user feedback size

\section{CONCLUSIONS}

This paper presents two strategies for construction of codebook for the processes of quantization in MU-MIMO-OFDM systems. Using zero forcing as technique of beamforming, the simulation results show that the simple codebook generation strategies based on scalar quantization are efficient, and they require smaller computational burden than other codebook generation algorithms based on higher dimensional vector quantization. Additionally, these simple scalar quantization strategies, by choosing an appropriate number of feedback bits, are also efficient for MIMO systems employing precoding techniques. 


\section{REFERENCES}

[1] D. J. Love, R. W. Health Jr., W. Santipach, and M. L. Honig, "What is the Value of Limited Feedback for MIMO Channels?॥, in IEEE Commun. Mag., vol. 42, no. 10, pp. 54-59, Oct. 2004

[2] C. K. Au-Yeung and D. J. Love, $\|$ On the Performance of Random Vector Quantization Limited Feedback Beamforming in a MISO Systeml, in IEEE Trans. Wireless Commun., vol. 6, no. 2, Feb. 2007.

[3] J. C. Roh, B. D. Rao, \|Transmit Beamforming in Multiple-Antenna Systems With Finite Rate Feedback: A VQ-Based Approach ॥, in IEEE Trans. on Information Theory, vol. 52, no. 3, March 2006.

[4] B. Khoshnevis and W. Yu, -Limited Feedback Multi-Antenna Quantization Codebook Design-Part I: Single-User Channels, ,Submitted to IEEE Transactions on Signal Processing, March2010.

[5] B. Khoshnevis and W. Yu, - Limited Feedback Multi-Antenna Quantization Codebook Design-Part II: Multiuser Channels,|ISubmitted to IEEE Transactions on Signal Processing, March 2010.

[6] D. J. Love, and R. W. Heath, —Limited feedback diversity techniques for correlated channels, ॥ IEEE Trans. Vehicular Tech., vol. 55, no. 2, pp. 718-722, Mar. 2006

[7] Part 11: Wireless LAN medium access control (MAC) and physical layer (PHY) specifications, ॥ IEEE Std 802.11ac/D4.0, Nov. 2012.

[8] Qi Wang, Hao Feng, Lenoard J. Cimini, Larry J. Greenstein, Douglas S. Chan and Ahmadreza Hedayat, "Comparison of Quantization Techniques for Downlink Multi-User MIMO Channels with Limited Feedback," IEEE Wireless Communications Letters, vol. 3, no. 1, pp. 165-168, Feb 2014.

[9] Q. Wang, H. Feng, L. J. Cimini, L. J. Greenstein, D. S. Chan, and A. Hedayat, -Sparse coding quantization for downlink MU-MIMO with limited channel state information feedback, $\|$ in Proc. 2013 IEEE MILCOM.

[10] N. Ravindran and N. Jindal, -Limited feedback-based block diagonalization for the MIMO broadcast channel,॥ IEEE J. Sel. Areas Commun., vol. 26, no. 8, pp. 1473-1482, Oct. 2008.

[11] W. Santipach, M.L. Honig -Optimization of Training and Feedback Overhead for Beamforming over Block Fading Channels\| IEEE Trans. Info. Theory, August 2009

[12] G. Caire, N. Jindal, M. Kobayashi, and N. Ravindran, Multiuser MIMO Achievable Rates with Downlink Training and Channel State Feedback, IEEE Trans. Information Theory, Vol. 56, No. 6, pp. 2845-2866, June 2010 